\title{
Spinacia oleracea L. extract attenuates hippocampal expression of TNF- $\alpha$ and IL-1 $\beta$ in rats exposed to chronic restraint stress
}

\author{
Sogand Pezeshki-Nia ${ }^{1}$, Masoumeh Asle-Rousta*2(D), Sanaz Mahmazi ${ }^{1}$ \\ Received: 13 Jun 2018 \\ Published: 24 Feb 2020
}

\begin{abstract}
Background: Restraint stress causes inflammation in nervous system that leads to emersion of neurodegenerative diseases. Spinach (Spinacia oleracea L.) contains different agents with antioxidant, antiapoptosis, and hepatoprotective properties. This study examined the effect of spinach hydroalcoholic extract (SHE) on TNF- $\alpha$ and IL-1 $\beta$ expression in hippocampus of male Wistar rats exposed to chronic restraint stress.

Methods: Rats were divided into 6 groups of 5: (1) control (intact); (2) nS-S200; (3) nS-S400; (4) stress; (5) stress-S200; (6) stressS400. Groups 2 and 3 and groups 5 and 6 received $S$. oleracea leaf hydroalcoholic extract in 200 and $400 \mathrm{mg} / \mathrm{kg}$ doses for 21 consecutive days by gavage. Groups 4, 5 and 6 were put in a restrainer 6 hours per day for 21 consecutive days. Then, the expression of IL-1 $\beta$ and TNF- $\alpha$ mRNAs and neuronal death in the hippocampus of rats were assessed by real time PCR and Nissl staining, respectively. Oneway analysis of variance was used for data analysis, and $p<0.05$ was considered statistically significant.

Results: The results showed that the expression of IL-1 $\beta$ and TNF- $\alpha$ was increased in hippocampus of rats exposed to stress compared to control groups $(\mathrm{p}<0.001)$. Furthermore, the expression of these proinflammatory cytokines was decreased in the stress-S200 and stress-S400 groups when compared to stress group $(\mathrm{p}<0.001)$. Immobility also caused neuronal death in CA1 region of hippocampus, and SHE reduced damage in CA1 pyramidal neurons layer in stressed rats.

Conclusion: Spinach decreases neuroinflammation in hippocampus of stressed rats, which may be due to its abundant antiinflammatory and antioxidant phytochemicals. The results of this study suggest that spinach may be effective in the prevention and treatment of neurodegenerative diseases.
\end{abstract}

Keywords: Spinacia oleracea L., IL-1 $\beta$, TNF- $\alpha$, Chronic restraint stress, Neuroinflammation.

Conflicts of Interest: None declared

Funding: None

*This work has been published under CC BY-NC-SA 1.0 license.

Copyright $₫$ Iran University of Medical Sciences

Cite this article as: Pezeshki-nia S, Asle-Rousta M, Mahmazi S. Spinacia oleracea L. extract attenuates hippocampal expression of TNF- $\alpha$ and IL-1 $\beta$ in rats exposed to chronic restraint stress. Med J Islam Repub Iran. 2020 (24 Feb);34:10. https://doi.org/10.47176/mjiri.34.10

\section{Introduction}

Stress is defined as physiological and psychological responses of the body to overcome changes in homeostasis. Although acute stresses are essential for survival, chronic stresses cause poor performance in the nervous and cardiovascular systems. Stress causes cognitive dysfunction and psychiatric disorders. The emergence and development of several neuropsychiatric diseases depend on stress $(1,2)$.

The most susceptible tissues responding to long-lasting

Corresponding author: Dr Masoumeh Asle-Rousta, mrousta@iauz.ac.ir

1. Department of Genetics, Zanjan Branch, Islamic Azad University, Zanjan, Iran

2. Department of Physiology, Zanjan Branch, Islamic Azad University, Zanjan, Iran stressors are hippocampus, amygdala, and prefrontal cortex that undergo functional and structural remodeling (3). Chronic stress selectively increases the number and activity of microglia in hippocampal CA3 region (4). Under chronic stress, microglia cells release interleukin-1 $\beta$ (IL-1 $\beta)$ and tumor necrosis factor $\alpha$ (TNF- $\alpha)(5)$. The elevation of proinflammatory cytokines production has been considered as a

$\uparrow$ What is "already known" in this topic:

Restraint stress induces oxidative stress, neuroinflammation, and neuronal apoptosis in hippocampus.

$\rightarrow$ What this article adds:

Hydroalcoholic extract of Spinacia oleracea L. suppresses IL-1 $\beta$ and TNF- $\alpha$ mRNA expression and reduces neuronal death in the hippocampal CA1 region in rats exposed to chronic restraint stress. 
critical factor for neurodegenerative diseases and psychiatric disorders (6).

It is generally accepted that stress causes inflammatory responses which contribute to cell damage and toxicity. Continuous excitation of neurons by ionotropic NMDA receptors in response to stress mediators (eg, cortisol) leads to the release of excitatory amino acids and could be the reason for cell death by excite-toxicity (7). Furthermore, hyperexcitation during stress generates reactive oxygen species (ROS) that could damage the cellular membrane. Damaged neuronal and glial cell release TNF- $\alpha$ and IL-1 $\beta$ that could induce neurodegenerative diseases $(8,9)$. In addition, high levels of glucocorticoids secreted by adrenal glands in response to stressful conditions cause neuronal damage, which is associated with increased production of ROS. Increasing the level of ROS in the CNS is also associated with neuropsychiatric disorders such as Alzheimer's disease, depression, and anxiety $(10,11)$.

Restraint stress has been used in rodents as a model for human disease and psychological disorders. Research studies have shown that restraint stress causes impairment in spatial memory's dependence on the hippocampus and hippocampal long-term potentiation. Synaptic loss and apical dendrites retraction in CA3 subregion of the hippocampus have been associated with such effects (12-15).

With respect to the role of neuroinflammation in neurodegeneration and cognitive impairment, it seems that preventing the production of inflammatory factors can greatly inhibit the development of neuropsychiatric diseases. Antioxidants are important in this regard because they largely prevent neuroinflammation (16). Herbs are being increasingly studied as important natural sources for antioxidant and anti-inflammatory substances. The flavonoids and phenolic compounds are effective substances that exert antiinflammatory and antioxidant activity $(17,18)$. S. oleracea L. that is rich in flavonoids, phenolic components, carotenoids, vitamins, and minerals has been used as an antioxidant and anti-inflammatory herb, hepatoprotective, and anticancer agent (19-21). Considering the effects of spinach in inflammation and oxidative stress, it is proposed that SHE protects hippocampus against chronic restraint stress induced inflammation. In this study, the SHE effects on the expression of IL- $1 \beta$ and TNF- $\alpha$ genes, as indicators of inflammation and neuronal death in hippocampus of rats exposed to chronic immobility stress, have been examined.

\section{Methods}

Chemicals: All chemicals (Chloral hydrate, Sodium citrate, EDTA, Ammonium sulfate) were purchased from Merck.

\section{Preparation of Spinacia oleracea L. extract}

S. oleracea L. was obtained from farms in Zanjan, Iran and verified by a specialist. Then, SHE was prepared from shade dried and powdered leaves: $100 \mathrm{~g}$ of pulverized $S$. oleracea was mixed with $300 \mathrm{~mL}$ of $70 \%$ ethanol, and the mixture was filtered by Whatman paper after 48 hours. Next, the extract was dried at $70^{\circ} \mathrm{C}$, stored at $4{ }^{\circ} \mathrm{C}$, and dissolved in distilled water to obtain desired concentration (22).

Animals and experimental design: Thirty male Wistar rats $(180-220 \mathrm{~g})$ were housed in the room temperature at $24^{\circ} \mathrm{C}$, with a 12 -hour light-dark cycle and fed with typical pellet food and water ad libitum. Rats were divided into 6 groups of 5: (1) control (no stress); (2) nS-S200; (3) nSS400; (4) stress; (5); stress-S200; and (6) stress-S400. Chronic stress was induced into groups 4,5 , and 6 by placing them in a restrainer 6 hours per day for 21 consecutive days (15). Groups 2 and 5 received $200 \mathrm{mg} / \mathrm{kg}$, and groups 3 and 6 received $400 \mathrm{mg} / \mathrm{kg}$ Spinacia extract per day by gavage during the 21 stress days. Control and stress groups received water instead of the extract by gavage. The doses of SHE were selected based on the effective doses in a study that proved the anxiolytic effect of spinach (22). The ethical committee of Islamic Azad University approved the study protocol.

\section{RNA extraction}

At the end of the experimental period, the rats were immediately sacrificed under chloral hydrate anesthesia. Brains were extracted from skull and hippocampi were collected immediately and kept in homemade RNAlater solution - for stabilization and protection of cellular RNA- (25 $\mathrm{mM}$ sodium citrate, $10 \mathrm{mM}$ EDTA and $70 \mathrm{~g}$ of ammonium sulfate/ $100 \mathrm{~mL}, \mathrm{pH} 5.2$ ) at $4^{\circ} \mathrm{C}$. Then, the total RNA was extracted from hippocampal tissues by RNX plus Kit (Sinaclon Co, Iran) according to the procedure.

\section{Real time PCR}

Two-step real time PCR was performed to analyze IL-1 $\beta$ and TNF- $\alpha$ RNA level in each sample. In the first step, cDNA was synthesized from $0.5 \mu \mathrm{g}$ of the total RNA using cDNA Synthesis Kit (TaKaRa) in a $20 \mu \mathrm{L}$ reaction mixture with random primers. In the second step, $1 \mu \mathrm{L}$ of this mixture was added to $24 \mu \mathrm{L}$ of PCR mixture containing each primer $(0.4 \mu \mathrm{M})$ and DNA polymerase master mix with SYBR Green (RealQ Plus Master Mix Green, Ampliqon). After heating at $95^{\circ} \mathrm{C}$ for 15 minutes, 40 cycles of PCRs were performed in 20 seconds at $95^{\circ} \mathrm{C}, 30$ seconds at $56^{\circ} \mathrm{C}$ and 30 seconds at $72^{\circ} \mathrm{C}$; and at the end of each cycle, the amount of fluorescence describing dsDNA was measured (Rotor-Gene Q, QIAGEN) (23). Primers were designed by Oligo7 software and checked by primer blast (https:/www.ncbi.nlm.nih.gov/tools/ primer-blast) to exclude unspecific primers. The sequences are presented in Table 1.

\begin{tabular}{lll}
\multicolumn{2}{l}{ Table 1. Designed primers for desired genes } \\
\hline Target gene & Forward: CAGCTTTCGACAGTGAGGAGA & Primer sequence $\left(5^{\prime}-3^{\prime}\right)$ \\
\hline IL-1 $\beta$ & Forward: ATGGGCTCCCTCTCATCAGT & Reverse: TTGTCGAGATGCTGCTGTGA \\
TNF- $\alpha$ & Forward: CGAGACCGATGTATATGCTTGC & Reverse: GTCCAGATGATTCAGAGCTCCA \\
B2M $*$ &
\end{tabular}




\section{Nissl Staining}

One brain hemisphere of each rat was embedded in paraffin, and 5 micrometer slices (from 2.7 to $3.7 \mathrm{~mm}$ posterior to bregma) were dyed by cresyl violet (1\%). Then, pyramidal neuron counting in CA1 was performed by optical microscope - zooming $\times 400$ - and neuronal death percentage was calculated [(average neuron number in sections of controls - number of neurons in sections of treated group) / average neuron number in controls sections $\times 100](15)$.

\section{Statistical analysis}

All data are shown as mean \pm standard error (SE). Significant differences between groups were examined using oneway analysis of variance following HSD and Tukey post hoc test. $\mathrm{P}<0.05$ was considered as statistically significant.

\section{Results}

Relative expression of IL- $1 \beta$ and TNF- $\alpha$ mRNA, compared to $\beta 2$ microglobulin mRNA as the reference gene, was examined by $\Delta \Delta \mathrm{Ct}$. The results of IL- $1 \beta$ mRNA expression data showed that mRNA level of this gene in stress group has been elevated $4.28( \pm 1.49)$ folds compared to control group. Treatment of both stress-S200 and stressS400 groups with defined doses of SHE reduced the IL-1 $\beta$ mRNA level to $1.77( \pm 0.4)$ and $0.89( \pm 0.1)$ folds of control, respectively. The changes of IL- $1 \beta$ mRNA level in SHE consumed groups were statistically significant $(p<0.000$ compared to stress group) (Fig. 1). However, treatments of non-stressed groups with counterpart doses of SHE did not make significant changes in IL-1 $\beta$ mRNA expression level compared to controls. The results also showed that immobilization caused a significant increase in the expression of TNF- $\alpha$ mRNA $8.05( \pm 2.50)$ fold of the control, and administration of SHE in both doses ameliorated stress effect in rats' hippocampus ( $p=0.005$ and $p<0.001)$ (Fig. 2). In addition, similar to the IL- $1 \beta$ gene in the hippocampus of nonstressed rats, the administration of SHE did not change the expression of TNF- $\alpha$ gene (Figs. 1 and 2).

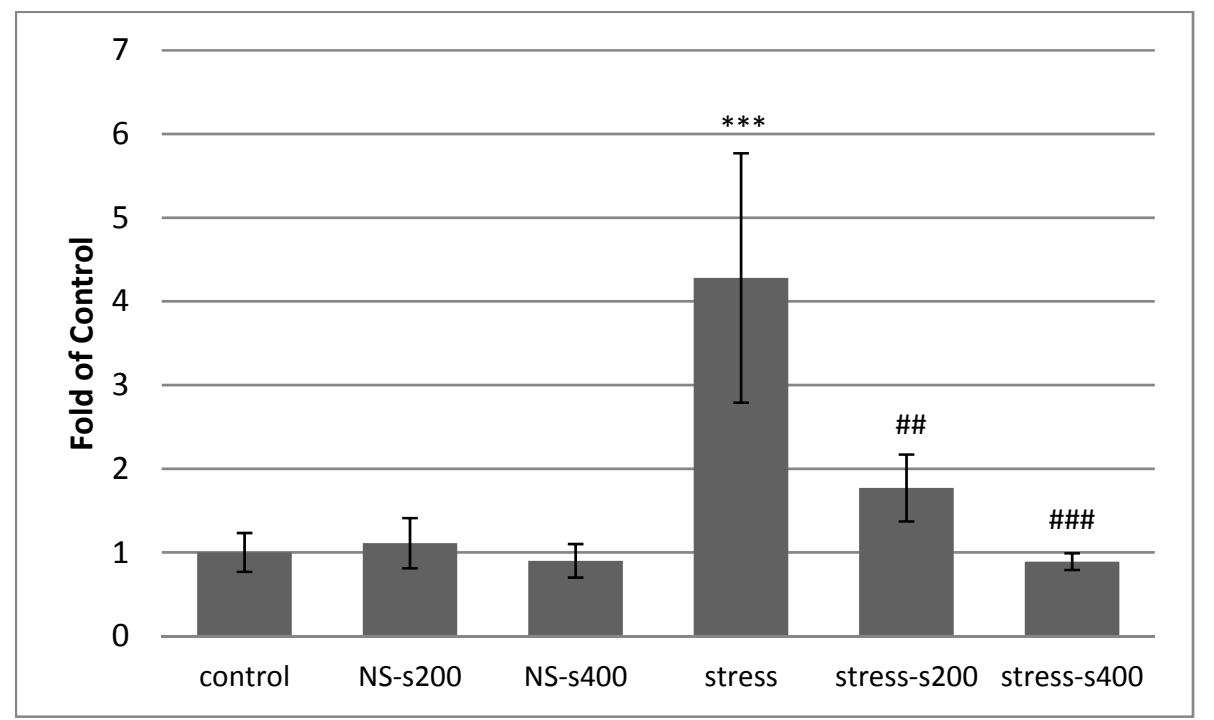

Fig. 1. The results of changes in the expression of TNF- $\alpha$ in the hippocampal tissue of chronic restraint stress rats. Values presented as mean $\pm \mathrm{SE}(\mathrm{n}=5)$. ${ }^{* * *} \mathrm{P}<0.001$ vs control; ${ }^{\# \#} \mathrm{P}<0.01$ and ${ }^{\# \#} \mathrm{P}<0.001$ vs stress group.

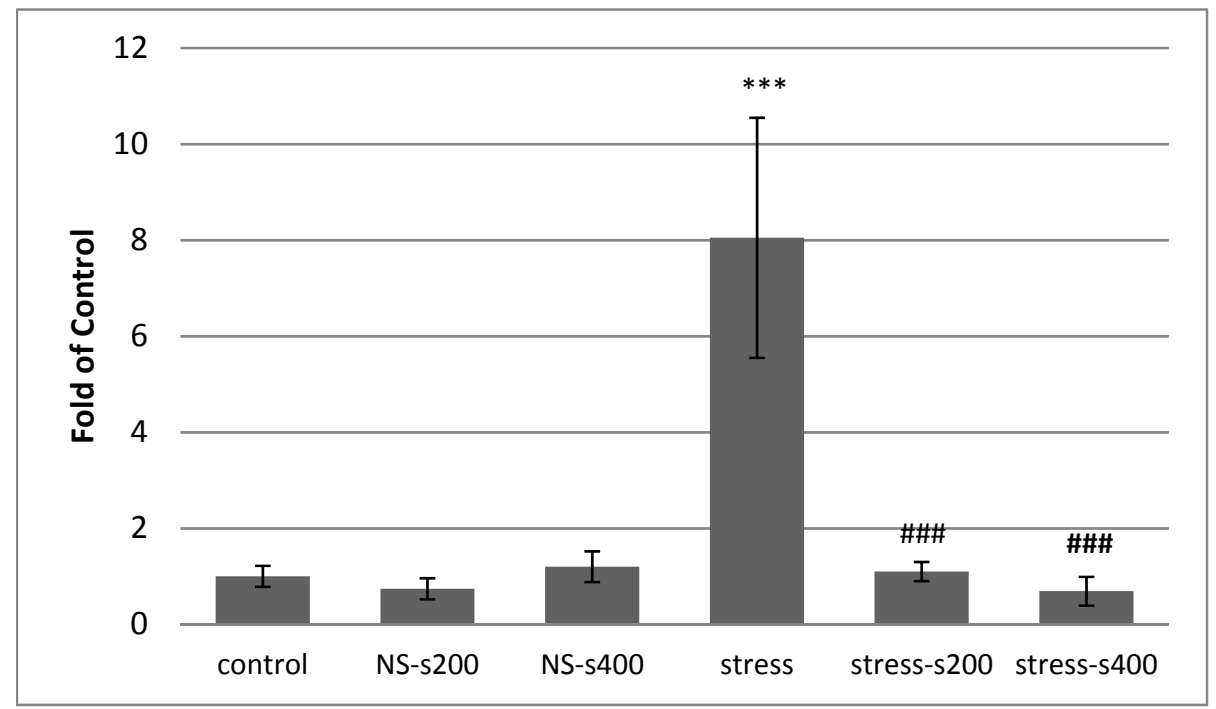

Fig. 2. The results of changes in the expression of IL-1 $\beta$ in the hippocampal tissue of chronic restraint stress rats. Values are presented as mean $\pm \mathrm{SE}(\mathrm{n}=5) .{ }^{* * *} \mathrm{P}<0.001$ vs control; ${ }^{\#} \mathrm{P}<0.001$ vs stress group. 


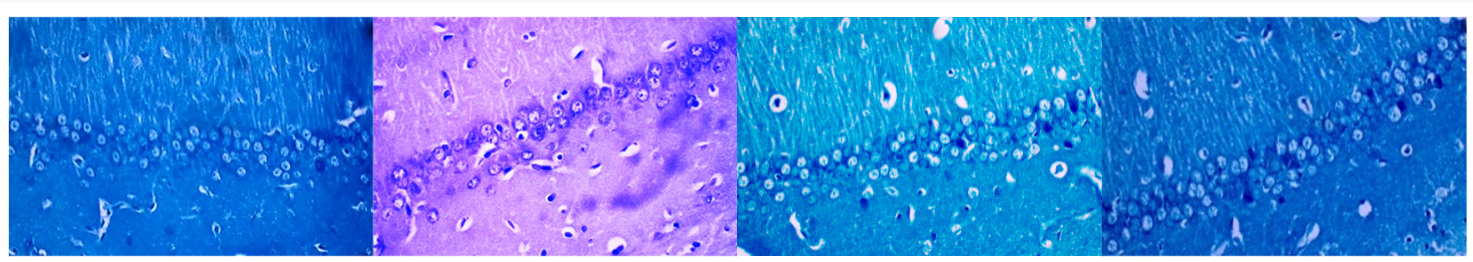

Fig. 3. Effect of chronic treatment of SHE on restraint-induced damage in CA1 region of rats' hippocampus. Photomicrographs showed a considerable neuronal loss and thinned pyramidal cells layer in CA1 area of the stress group and a significant improvement in rats treated with SHE. Photos represent $\times 400$ magnification. Left to right: control, stress, stress-S200 and stress-S400.

Table 2. Effect of chronic treatment of SHE on immobility-induced neuronal loss in CA1 region of hippocampus.

\begin{tabular}{lc}
\hline Group & Percentage of neuronal loss in CA1 area (mean \pm SEM) \\
\hline Control & 0 \\
nS-S200 & $1.18 \pm 0.49$ \\
nS-S400 & $1.33 \pm 0.80$ \\
Stress & $11.56 \pm 1.98 * * *$ \\
Stress-S200 & $4.45 \pm 1.35$ \\
Stress-S400 & $4.79 \pm 0.84^{\# \#}$ \\
\hline The percentage of neuronal loss was calculated [(average neuron number in control sections - neuron number in treated group sections)/average neuron
\end{tabular}

The percentage of neuronal loss was calculated [(average neuron number in control sections-neuron number in treated group sections)/

Reduction in neuronal layer thickness and number of pyramidal neurons in CA1 area were observed by a histological study (Fig. 3). Restraint stress reduced the pyramidal neurons population in CA1 $(11.56 \pm 0.62 \%)(\mathrm{p}<0.001)$ (Table 1). SHE prevented neuronal reduction in CA1 and the neuronal death percentage in this region in stress-S200 and stress-S400 groups compared to Stress group $(\mathrm{p}<0.001)$ (Table 2). No remarkable change was observed in nS-S200 and nS-S400 groups.

\section{Discussion}

In general, stress affects the central nervous system and can exacerbate the effects of stress factors, which may cause abnormal responses to many neurological and physiological problems $(1,24)$. In the hippocampus, stress can cause dendritic retraction of pyramidal neurons and reduction in synapse number (25). Such events are due to the change in the production and secretion of cytokines that lead to memory impairment, Alzheimer's disease, and neuropsychiatric disorders $(26,27)$. Previously, it was shown that chronic restraint stress in rats leads to spatial memory impairment and anxiety behavior $(15,22)$. The results of this study confirmed that the proinflammatory factors TNF$\alpha$ and IL- $1 \beta$ expression were increased in the hippocampus of rats exposed to restraint stress 6 hours a day for 21 consecutive days, which was followed by neuronal death in CA1 area of hippocampus. This finding is in agreement with those of other studies that have demonstrated restraint or immobilization stress upregulates the expression of IL$1 \beta$ and TNF- $\alpha$ in brain's compartments and causes neuronal death in hippocampus $(15,28,29)$. For example (regarding to IL- $1 \beta$ ), it has been reported that IL- $1 \beta$ and TNF- $\alpha$ genes expression increased in animal models of restraint stress in hippocampus (29). Moreover, a chronic restraint stress in the condition similar to this study ( $6 \mathrm{~h}$ a day for 21 successive days) demonstrated that TNF- $\alpha$ level increased in the hippocampus of stressed rats at the end of restraint period (30).

Elevation of IL-1 $\beta$ in stressed mice induces apoptosis as a result of ROS production (31). Thus, considering the role of IL- $1 \beta$ and TNF- $\alpha$ in the onset of apoptosis, neuronal reduction in hippocampal neurons is also reasonable. Accordingly, an increase in the level of IL- $1 \beta$ and TNF- $\alpha$ leads to neurodegeneration and cognitive impairment, but prevention of inflammation factors expression may inhibit its neuropsychiatric effects $(32,33)$.

Spinacia oleracea, which is known as spinach and is consumed as vegetable, contains many flavonoids, phenolic compounds, carotenoids, vitamins, and minerals and have significant anti-inflammatory and antioxidant roles (1921). The investigation of the inhibitory effects of $S$. oleracea hydroethanolic extract on inflammation and neuronal death in the hippocampus of chronic restraint-stressed rats indicated the amelioration of IL- $1 \beta$ and TNF- $\alpha$ expression and suppression of cell death in hippocampus compared to the stress group $(\mathrm{p}<0.05$ and $\mathrm{p}<0.001$, respectively). Also, it has been demonstrated that intraperitoneally injection of $S$. oleracea leaf extract $(50 \mathrm{mg} / \mathrm{kg})$ causes inhibition of IL- $1 \beta$ and TNF- $\alpha$ expression in the brain of lipopolysaccharide injected mice (34).

Various studies have been conducted on the antioxidant and anti-inflammatory effects of the active constituents of spinach. Although the antioxidant impact of spinach has been widely considered in neurodegeneration diseases, other neuroprotective aspects of spinach should also be noted. Pervasive studies have shown reciprocal impacts of oxidative stress and inflammation pathways (35). Therefore, in addition to antioxidant activity, the neuroprotective effect of spinach may associate with anti-inflammatory impacts of its phytochemicals.

Luteolin and apigenin, flavonoids found in spinach, inhibit the induction of TNF- $\alpha$ and IL-6 in microglia by inhibiting STAT-1 (36). In addition, Quercetin, another flavonoid found in spinach, reduces TNF- $\alpha$ and IL- $1 \beta$ production in macrophage in rats (37). In another study, quercetin, kaempferol, and genistein (spinach flavonoids) showed anti-inflammatory effects by inhibition of NF- $\mathrm{kB}$, iNOS, and STAT-1 in signaling pathways (38). Youngha et al (2011) demonstrated that beta-carotene, a carotenoid found in spinach, could suppress the expression of proinflamma- 
tory factor IL- 8 by preventing NF- $\kappa \mathrm{B}$ activation (39). Besides, several studies have shown that vitamin $\mathrm{E}$, which is abundantly found in spinach, reduces lipid peroxidation and production of superoxide, preventing TNF- $\alpha$, IL- $1 \beta$ and IL-6 expression (40). Therefore, SHE, which is rich in anti-inflammatory and antioxidant compounds, has been able to reduce the expression of TNF- $\alpha$ and IL- $1 \beta$ genes and inhibit CA1 area neuronal death, which is in agreement with the results of the present study. Recently, the results of a study suggested that hydroalcoholic extract of spinach in the same doses used in the present study improved spatial memory in rats exposed to immobility stress (22). Such results indicate the probability of the memory protecting effect of spinach by preventing the expression of inflammatory factors.

The results of this study also showed that $S$. oleracea L. extract does not influence the expression of IL-1 $\beta$ and TNF$\alpha$ in rats that had not been exposed to stress earlier. This constancy in the cytokine's expression may be due to the presence of hemostasis in the hippocampus of non-stressed, rats which preserves the antioxidant/oxidant balance. Despite the fact that hemostasis is missed in rats that had been exposed to stress, active constituents of spinach are used to maintain hemostasis. Neuroinflammation is closely linked to oxidative stress, which is the main mechanism of stressrelated disorders, especially in the brain (41).

\section{Conclusion}

Hydroalcoholic extract of $S$. oleracea reduces neuroinflammation and hippocampal neuronal death in stressed rats, which may be due to its anti-inflammatory and antioxidant phytochemicals. Neuroinflammation and neural apoptosis are common symptoms of neurodegeneration, so it seems that spinach can be effective in preventing neurodegenerative diseases by reducing neuroinflammation and neuronal death.

\section{Acknowledgments}

The authors thank Masoumeh Tarasi and Afsaneh Nazari for technical assistance.

\section{Conflict of Interests}

The authors declare that they have no competing interests.

\section{References}

1. McEwen BS, Bowles NP, Gray JD, Hill MN, Hunter RG, Karatsoreos IN, et al. Mechanisms of stress in the brain. Nature Neurosci. 2015;18(10):1353.

2. Saleh A, Potter GG, McQuoid DR, Boyd B, Turner R, MacFall JR, et al. Effects of early life stress on depression, cognitive performance and brain morphology. Psychol Med. 2017;47(1):171-81.

3. McEwen BS, Nasca C, Gray JD. Stress effects on neuronal structure: hippocampus, amygdala, and prefrontal cortex. Neuropsychopharmacol. 2016;41(1):3.

4. Tynan RJ, Naicker S, Hinwood M, Nalivaiko E, Buller KM, Pow DV, et al. Chronic stress alters the density and morphology of microglia in a subset of stress-responsive brain regions. Brain Behav Immun. 2010;24(7):1058-68.

5. Calcia MA, Bonsall DR, Bloomfield PS, Selvaraj S, Barichello T, Howes OD. Stress and neuroinflammation: a systematic review of the effects of stress on microglia and the implications for mental illness Psychopharmacol. 2016;233(9):1637-50.

6. Hong H, Kim BS, Im HI. Pathophysiological role of neuroinflammation in neurodegenerative diseases and psychiatric disorders. Int Neurol J. 2016;20(Suppl 1):S2.

7. Kubera M, Obuchowicz E, Goehler L, Brzeszcz J, Maes M. In animal models, psychosocial stress-induced (neuro) inflammation, apoptosis and reduced neurogenesis are associated to the onset of depression. Prog Neuro Psychoph. 2011 Apr 29;35(3):744-59.

8. Olmos G, Lladó J. Tumor necrosis factor alpha: a link between neuroinflammation and excitotoxicity. Mediat Inflamm. 2014:1-13.

9. Réus GZ, Fries GR, Stertz L, Badawy M, Passos IC, Barichello T, et al. The role of inflammation and microglial activation in the pathophysiology of psychiatric disorders. Neuroscience. 2015;300:141-54.

10. Juszczak GR, Stankiewicz AM. Glucocorticoids, genes and brain function. Prog Neuro Psychoph. 2018;82:136-68.

11. Fung L, Hardan A. Oxidative Stress in Psychiatric Disorders. In The Therapeutic Use of N-Acetylcysteine (NAC) in Medicine 2019 (pp. 53-72). Adis, Singapore.

12. Campos AC, Fogaca MV, Aguiar DC, Guimaraes FS. Animal models of anxiety disorders and stress. Braz J Psychiatary. 2013;35:S101-11.

13. Wang M, Ramasamy VS, Samidurai M, Jo J. Acute restraint stress reverses impaired LTP in the hippocampal CA1 region in mouse models of Alzheimer's disease. Sci Rep. 2019;9(1):10955.

14. McEwen BS. Stress-induced remodeling of hippocampal CA3 pyramidal neurons. Brain Res. 2016;1645:50-4.

15. Bigdeli Y, Asle-Rousta M, Rahnema M. Effects of Limonene on Chronic Restraint Stress-Induced Memory Impairment and Anxiety in Male Rats. Neurophysiology. 2019;51(2):107-13.

16. Shal B, Ding W, Ali H, Kim YS, Khan S. Anti-neuroinflammatory potential of natural products in attenuation of Alzheimer's disease. Front Pharmacol. 2018;9:548-65.

17. Spencer JP, Vafeiadou K, Williams RJ, Vauzour D. Neuroinflammation: modulation by flavonoids and mechanisms of action. Mol Aspects Med. 2012;33(1):83-97.

18. Rangarajan P, Karthikeyan A, Dheen ST. Role of dietary phenols in mitigating microglia-mediated neuroinflammation. Neuromol Med. 2016;18(3):453-64.

19. Roberts JL, Moreau R. Functional properties of spinach (Spinacia oleracea L.) phytochemicals and bioactives. Food Funct. 2016;7(8):3337-53.

20. Jain NK, Singhai AK. Ameliorative effects of Spinacia oleracea L. seeds on carbon tetrachloride $(\mathrm{CCl} 4)$-induced hepatotoxicity: in vitro and in vivo studies. Asian Pac J Trop Biomed. 2012;2(1):S232-7.

21. Nagar A, Shukla AK, Bigoniya P. Anti-inflammatory potential of Spinacia oleracea leaf extract. J Nat Pharm. 2011;2(2):80-87.

22. Tarasi M, Asle-Rousta M. Effect of spinach (Spinacia oleracea L.) extract on chronic restraint stress-induced memory deficit and anxiety in male rats. Razi J Med Sci. 2018;25(169):48-54. (Persaian)

23. Enayati S, Seifirad S, Amiri P, Abolhalaj M, Mohammad-Amoli M. Interleukin-1 beta, interferon-gamma, and tumor necrosis factor-alpha gene expression in peripheral blood mononuclear cells of patients with coronary artery disease. ARYA Atheroscler. 2015;11(5):267-74.

24. Frank MG, Weber MD, Watkins LR, Maier SF. Stress-induced neuroinflammatory priming: a liability factor in the etiology of psychiatric disorders. Neurobiol Stress. 2016;4:62-70.

25. Tomar A, Polygalov D, Chattarji S, McHugh TJ. The dynamic impact of repeated stress on the hippocampal spatial map. Hippocampus. 2015;25(1):38-50.

26. Hanson JL, Nacewicz BM, Sutterer MJ, Cayo AA, Schaefer SM, Rudolph KD, et al. Behavioral problems after early life stress: contributions of the hippocampus and amygdala. Biol Psychiatry. 2015;77(4):314-23.

27. Pfau ML, Russo SJ. Neuroinflammation regulates cognitive impairment in socially defeated mice. Trends Neurosci. 2016;39(6):353-5.

28. Tan S, Wang Y, Chen K, Long Z, Zou J. Ketamine alleviates depressive-like behaviors via down-regulating inflammatory cytokines induced by chronic restraint stress in mice. Biol Pharm Bull. 2017;40(8):1260-7.

29. Sathyanesan M, Haiar JM, Watt MJ, Newton SS. Restraint stress differentially regulates inflammation and glutamate receptor gene expression in the hippocampus of $\mathrm{C} 57 \mathrm{BL} / 6$ and $\mathrm{BALB} / \mathrm{c}$ mice. Stress. 2017;20(2):197-204.

30. Azadbakht AA, Radahmadi M, Javanmard SH, Reisi P. The effects of doxepin on stress-induced learning, memory impairments, and 
TNF- $\alpha$ level in the rat hippocampus. Res Pharm Sci. 2015;10(5):460 5 .

31. Kempuraj D, Thangavel R, Natteru PA, Selvakumar GP, Saeed D, Zahoor $\mathrm{H}$, et al. Neuroinflammation induces neurodegeneration. J Neurol Neurosurg Spine. 2016;1(1):1003.

32. Clark IA, Vissel B. Neurodegenerative disease treatments by direct TNF reduction, SB623 cells, maraviroc and irisin and MCC950, from an inflammatory perspective-a Commentary. Expert Rev Neurother. 2019;19(6):535-43.

33. McFarland AJ, Davey AK, McDermott CM, Grant GD, Lewohl J, Anoopkumar-Dukie S. Differences in statin associated neuroprotection corresponds with either decreased production of IL- $1 \beta$ or TNF- $\alpha$ in an in vitro model of neuroinflammation-induced neurodegeneration. Toxicol Appl Pharmacol. 2018;344:56-73.

34. Sharma N, Kapoor M, Nehru B. Spinacea oleracea L. extract protects against LPS induced oxidative stress, inflammatory response and ensuing biochemical, neurochemical and neurobehavioral impairment in mice. Int J Pharm Pharm Sci. 2014;6:203-10.

35. Leszek J, E Barreto G, Gasiorowski K, Koutsouraki E, Aliev G. Inflammatory mechanisms and oxidative stress as key factors responsible for progression of neurodegeneration: role of brain innate immune system. CNS Neurol Disord Targets. 2016;15(3):329-36.

36. Rezai-Zadeh K, Ehrhart J, Bai Y, Sanberg PR, Bickford P, Tan J, et al. Apigenin and luteolin modulate microglial activation via inhibition of STAT1-induced CD40 expression. J Neuroinflammation. 2008;5(1):41:1-10.

37. Li Y, Yao J, Han C, Yang J, Chaudhry MT, Wang S, et al. Quercetin, inflammation and immunity. Nutrients. 2016;8(3):167-181.

38. Hämäläinen M, Nieminen R, Vuorela P, Heinonen M, Moilanen E. Anti-inflammatory effects of flavonoids: genistein, kaempferol, quercetin, and daidzein inhibit STAT-1 and NF- $\kappa$ B activations, whereas flavone, isorhamnetin, naringenin, and pelargonidin inhibit only NF- $\kappa \mathrm{B}$ activation along with their inhibitory effect on ihibitory effect on iNOS expression and NO production in activated macrophages. Mediators Inflamm. 2007:1-10.

39. Youngha KIM, Seo JH, Hyeyoung KIM. $\beta$-Carotene and lutein inhibit hydrogen peroxide-induced activation of NF- $\mathrm{BB}$ and IL-8 expression in gastric epithelial AGS cells. J Nutr Sci Vitaminol (Tokyo). 2011;57(3):216-23.

40. Bo L, Jiang S, Xie Y, Kan H, Song W, Zhao J. Effect of vitamin E and omega-3 fatty acids on protecting ambient PM2. 5-induced inflammatory response and oxidative stress in vascular endothelial cells. PLoS One. 2016;11(3):e0152216.

41. Patki G, Solanki N, Atrooz F, Allam F, Salim S. Depression, anxietylike behavior and memory impairment are associated with increased oxidative stress and inflammation in a rat model of social stress. Brain Res. 2013;1539:73-86. 